\title{
Can gamma-ray bursts constrain quintessence?
}

\author{
Tristano Di Girolamo $\nmid \S$ Riccardo Catena $\ddagger$ Mario Vietri $\ddagger$ and \\ Giuseppe Di Sciascio $†$ \\ $\dagger$ Istituto Nazionale di Fisica Nucleare, Sezione di Napoli, Complesso Universitario di \\ Monte Sant'Angelo, Via Cintia, 80126 Napoli, Italy \\ $\ddagger$ Scuola Normale Superiore, Piazza dei Cavalieri, 56100 Pisa, Italy
}

\begin{abstract}
Using the narrow clustering of the geometrically corrected gamma-ray energies released by gamma-ray bursts, we investigate the possibility of using these sources as standard candles to probe cosmological parameters such as the matter density $\Omega_{m}$ and the cosmological constant energy density $\Omega_{\Lambda}$. By simulating different samples of gamma-ray bursts, we find that $\Omega_{m}$ can be determined with accuracy $\sim 7 \%$ with data from 300 sources. We also show that, if $\Omega=1$ is due to a quintessence field, some of the models proposed in the literature may be discriminated from a Universe with cosmological constant, by a similar-sized sample of gamma-ray bursts.

PACS numbers: 98.70.Rz, 98.80.Es
\end{abstract}

\section{Introduction}

Recent studies have pointed out that Gamma-Ray Bursts (GRBs) may be used as standard cosmological candles. The prompt $\gamma$-ray energy release, when neglect is made of the conical geometry of the emission, spans nearly three orders of magnitude, and the distribution of the opening angles of the emission, as deduced from the timing of the achromatic steepening of the afterglow emission, spans an identically wide range of values. However, when the apparently isotropic energy release and the conic opening of the emission are combined to infer the intrinsic, true energy release, the resulting distribution does not widen, as is expected for uncorrelated data, but shrinks to a very well determined value (Frail et al. 2001; Panaitescu \& Kumar 2001; Bloom, Frail, \& Kulkarni 2003), with a remarkably small (one-sided) scattering, corresponding to about a factor of 2 in total energy. Similar studies in the X-ray band (Piran et al. 2001; Berger, Kulkarni, \& Frail 2003) have reproduced the same results.

It is thus very tempting to study to what extent this property of GRBs makes them suitable cosmological standard candles. After an early investigation made by Cohen \& Piran (1997), Schaefer (2003) proposed using two well known correlations of the GRBs luminosity (with variability, and with time delay) to the same end, while Dai, Liang, \& Xu (2004) and Ghirlanda et al. (2004) exploited the recently reported relationship

$\S$ To whom correspondence should be addressed (tristano@na.infn.it) 
between the beaming-corrected $\gamma$-ray energy and the local observer peak energy in GRBs (Ghirlanda, Ghisellini, \& Lazzati 2004). We instead neglect these three relationships and concentrate on the very narrow spread of the true, geometrically corrected energy release as a distance indicator, recalling however that its determination for any given bursts requires substantially more information than the methods presented by Schaefer, and the other authors mentioned above.

As for the possible variation of ambient density from burst to burst, which may widen the distribution of bursts energies, Frail et al. (2001) remarked that this spread is already contained in their data sample, and yet the distribution of energy releases is still very narrow. If we were somehow able to measure the distribution ambient densities, and subtract these from the sample, the distribution of energy releases should narrow even more, not widen: in fact, since we obviously expect the two distributions to be uncorrelated, we also expect the one resulting from their combination to be wider than the intrinsic distribution of energy releases.

There are at least two respects in which GRBs are better than type Ia SuperNovae (SNIa) as cosmological candles, one in which they are worse, and one in which they are probably even. On the one hand, GRBs are easy to find and locate: even 1980s' technology allowed BATSE to locate $\sim 1$ GRB per day, despite an incompleteness of about $1 / 3$, making the build-up of a 300-object database a one-year enterprise, with old technology. The launch of the Swift satellite, which took place on 20 November 2004, is expected to detect GRBs at about the same rate as BATSE, but with a nearly perfect capacity for identifying their redshifts simultaneously with the afterglow observations $\|$. Second, GRBs have been detected out to very high redshifts: even the current sample of about 40 objects (Greiner 2004) contains several events with $z>3$, with an absolute maximum of $z=4.5$ for GRB 000131. This should be contrasted with the difficulty of locating SNe with $z>1$, and the absolute lack of any SN with $z>2$. The currently observed distribution of GRBs redshifts contains instead 21 events with $z>1$ out of a total of 39 (see Figure 4).

On the other hand, the distribution of luminosities of SNIa is narrower than the distribution of GRBs energy releases, corresponding to a magnitude dispersion $\sigma_{M}=0.18$ rather than $\sigma_{M}=0.75$. However, the two break even (probably) in terms of our understanding of the underlying physical reasons for the uniformity of the distributions, which is wanting in both cases.

Thus GRBs may provide a complementary standard candle, out to distances which cannot be probed by SNIa, their major limitation being the larger intrinsic scatter of the energy release, as compared to the small scatter in peak luminosities of SNIa. It is thus important to assess whether this larger scatter still allows GRBs to be used as standard candles. To this end, and as a first aim of the paper, we carry out numerical simulations of random samples of GRBs, whose energy releases are distributed as found out by Frail et al. (2001), to see to what extent global cosmological parameters can be identified by || http://swift.gsfc.nasa.gov/docs/swift/proposals/appendix_f.html 
an arbitrarily large (but within reason) sample of hypothetical observations.

As a second aim of the paper, we study, also by means of simulations, whether the larger redshift range spanned by GRBs, when compared with SNIa, allows us to identify specific models for quintessence. If the non-matter component of the overall energy density in the Universe were indeed a constant, at $z \approx 1$ the increase in the matter content would dwarf it, and there would be no difference, at larger redshifts, between a model with cosmological constant, and one without (we call this the null hypothesis). However, if the cosmological constant is not constant at all, but is provided by the new heuristic field called quintessence, one may hope that at least some models display evolution of the cosmological distances (luminosity, fluence, angular, and so on), which differ substantially from those of the model with cosmological constant. It is thus our second aim to study universes with different, simple models for quintessence, to see whether GRBs observations may be able to discriminate between them. In other words, we study whether GRBs can reject the hypothesis of a constant $\Lambda$.

We stress that this paper is not aimed at displaying the potential for cosmological investigation by any coming satellite, but instead at determining whether the size of a realistically obtainable set of data (perhaps to be obtained by means of a dedicated satellite) is useful for cosmological studies. We assume that we know, for every burst in our sample, the redshift, and the opening-corrected apparent fluence (i.e., the apparent luminosity integrated over the burst duration), and that there is no evolution with redshift of the bursts intrinsic energy release. We remark that it is not necessary to have a complete and homogeneous sample of objects to carry out this exercise, and that the precise value of the bursts average energy release is not necessary, because as usual in cosmological tests, we are fitting the dependence of the luminosity distance upon redshift and cosmological parameters, not its absolute normalization.

The plan of the paper is as follows. In Section 2, we display a simulation with the simple aim of showing the power of a set of 300 GRBs distributed out to large redshifts, in rejecting or accepting the presence of a cosmological constant term in the Universe density distribution. A test like this would also be useful in practice, since it would be completely independent of observations of fluctuations in the Cosmic Microwave Background Radiation (CMBR). Then, in Section 3, we assume a $\Omega_{\Lambda}=0, \Omega_{m}=1$ cosmology, and test the ability of similar-size sets of GRBs to determine $\Omega_{m}=1$. In Section 4 , we assume instead $\Omega_{\Lambda} \neq 0, \Omega_{m}+\Omega_{\Lambda}=1$, and test the ability of the same samples of GRBs to identify the correct values of $\Omega_{m}$. In Section 5 , we abandon the hypothesis that $\Lambda$ is a constant, and turn to different quintessence models, showing that at least one of the important ones (Gasperini, Piazza, \& Veneziano 2002) can be easily discriminated from the others, and from the null hypothesis. In Section 6, we summarize and conclude. 


\section{A simple test}

First, in order to show what we are aiming at, we performed a Kolmogorov-Smirnov (KS) test on two data sets made of 300 GRBs simulated in two different cosmological models, one with $\Omega_{m}=1$ and $\Omega_{\Lambda}=0$ and the other with $\Omega_{m}=0.3$ and $\Omega_{\Lambda}=0.7$, but both with a Hubble constant $H_{0}=65 \mathrm{~km} \mathrm{~s}^{-1} \mathrm{Mpc}^{-1}$ (as it will be assumed throughout the paper). We preferred the KS test to others since it is applicable to any kind of continuous distribution that is a function of a single independent variable, which is the case we are dealing with. The $\chi^{2}$ test, for comparison, is more suited to point out differences between binned distributions. For the KS test each list of data points, after ordering, is converted to a cumulative distribution function giving the fraction of data points to the left of a given value for the variable. Then the maximum value $D$ of the absolute difference between these two cumulative distribution functions is adopted as the test statistic, and the probability $Q_{K S}$ of finding values greater than the observed $D$ gives the significance level for the null hypothesis that the data sets are drawn from the same distribution (Von Mises 1964).

We assume that GRBs are indeed standard candles with true $\gamma$-ray energy released, $E_{\gamma}$, following a Gaussian distribution in its logarithm with mean $\mu=51.1$ (if $E_{\gamma}$ is expressed in erg units, Bloom, Frail, \& Kulkarni 2003) and $\sigma=0.3$ (corresponding to a multiplicative factor of 2), and that they are distributed in the Universe according to the model of star formation rate $R_{S F 1}(z)$ reported in Porciani \& Madau (2001), which matches the $\log N-\log P$ relation (GRB number counts vs. peak photon flux) obtained with BATSE data. Applying the KS test to the redshift distributions, we found that the probability that the two data sets are the same is $Q_{K S}=0.031$, a "no man's land" value for this test. On the other hand, the application of the KS test to the parameter $\log d_{L}^{2}(z)$, where $d_{L}(z)$ is the luminosity distance, resulted in a significant probability $Q_{K S} \sim 10^{-14}$, which tells us that it is possible to discriminate between the two different cosmological models if a set of 300 GRB luminosity distances is known, without any reference to CMBR data.

\section{Simulations in a $\Lambda=0$ cosmology}

We consider now a $\Lambda=0$ cosmology, in which the only contribution to the density parameter is given by $\Omega_{m}$. We assume for GRBs the same energy distribution as for the KS test. However, the assumed mean value is not relevant for our investigation, since it is the dispersion value that constrains the cosmological density parameter. The dispersion of the $\gamma$-ray energy released in GRBs may be pinned down in the future by a local sample of sources, such as the recently discovered GRB 030329 and 031203, at $z=0.1685$ (Price et al. 2003) and $z=0.1055$ (Malesani et al. 2004) respectively (see Section 5 for a more detailed discussion on this point).

The standard candle energy is related to the fluence of the burst $f_{\gamma}=E_{\gamma}(1+$ 
$z) /\left(4 \pi d_{L}^{2}(z)\right)$ via the luminosity distance $d_{L}(z)$, whose expression for $\Lambda=0$ is:

$$
d_{L}(z)=\frac{c}{H_{0}} \frac{2\left[2-\Omega_{m}+\Omega_{m} z-\left(2-\Omega_{m}\right) \sqrt{1+\Omega_{m} z}\right]}{\Omega_{m}^{2}}
$$

Since the $k$-correction is independent of any cosmological parameter, we take no account of it. A discussion about its effects on the distribution of GRBs energy releases is made in Bloom, Frail, \& Sari (2001). In order to have a linear propagation of errors throughout our simulations, we choose to construct with GRBs a Hubble diagram $\log d_{L}^{2}-z$, since the distribution of the parameter $\log d_{L}^{2}$ is the same of that of $\log E_{\gamma}$, and therefore it is Gaussian.

The number of GRBs per redshift unit is given by the expression:

$$
\frac{d N_{G R B}}{d z}=\frac{n(z)(d V / d z)}{\int_{0}^{z_{\max }} n(z)(d V / d z) d z}
$$

where $n(z)$ is the redshift distribution function, extending to the maximum redshift for GRB explosions $z_{\max }$, and $d V / d z$ is the comoving volume element, which for $\Lambda=0$ is:

$$
\frac{d V}{d z}=\frac{c}{H_{0}} \frac{4 \pi d_{L}^{2}(z)}{(1+z)^{2}\left[\Omega_{m}(1+z)^{3}+\left(1-\Omega_{m}\right)(1+z)^{2}\right]^{1 / 2}}
$$

As for the KS test, we assume that the redshifts of GRBs are distributed according to the model of star formation rate $R_{S F 1}(z)$ reported in Porciani \& Madau (2001):

$$
n(z) \propto \frac{\exp (3.4 z)}{\exp (3.8 z)+45}
$$

This function increases rapidly between $z=0$ and 1 , peaks between $z=1$ and 2 , and gently declines at higher redshifts. We fix $z_{\max }=5$.

In order to study the ability of GRBs in probing the cosmological parameters as a function of their number, we have simulated different samples with $N_{G R B}=10,30$, 100, 300 and 1000. Moreover, in order to be free from statistical fluctuations, we have performed $10^{2}, 10^{3}$, and $10^{4}$ realizations of each of these samples.

Now the simulation of a GRB consists of the random sampling of both the redshift $z$ and the true $\gamma$-ray energy released $E_{\gamma}$, according to the respective adopted distributions. Given a cosmological model, from these coupled values we obtain the corresponding value for the parameter $\log d_{L}^{2}$, which we plot on the Hubble diagram as a function of $z$. At this point we perform a $\chi^{2}$ minimization of the simulated data to see with what accuracy the fit reproduces the input cosmology. The measurement error on $\log d_{L}^{2}$ is assumed to be $\sigma=0.3$.

In Table 1 the mean results of our repeated fits are reported for an input cosmology with $\Omega_{m}=1$. First, from this Table it is evident that the mean values obtained from the fits are independent of the number of sample realizations, i.e., the intrinsic fluctuations corresponding to different samples of GRBs in the same cosmological model, are small. Moreover, the Table shows how the accuracy of GRBs in constraining the matter density fraction $\Omega_{m}$ increases with their number $N_{G R B}$. The given cosmology is readily reproduced by the best fit value for any $N_{G R B}$, while its dispersion is reduced 
from $\sim 30 \%$ for a sample with $N_{G R B}=10$ to $\sim 3 \%$ for $N_{G R B}=1000$. We have also carried out simulations for different values of $\Omega_{m}$, but still $\Omega_{\Lambda}=0$, obtaining every time very similar results.

It is worth noting that it will be very difficult and time consuming to determine $E_{\gamma}$ of 300 GRBs to the accuracy required. Even then the resulting $7 \%$ error on $\Omega_{m}$ is larger than the $\sim 1 \%$ errors today from WMAP and, eventually, SNAP. Still, an independent measurement of a parameter of such paramount importance need not be useless, even if late in coming.

\section{Simulations in a $\Lambda$-dominated cosmology}

We move now to a $\Lambda$-dominated cosmology, in which the contributions to the density parameter are given by the mass density, $\Omega_{m}$, and by the cosmological constant energy density, $\Omega_{\Lambda}$. In the light of the recent observations of the cosmic microwave background anisotropy (Bennett et al. 2003), we restrict our simulations to a flat Universe $\Omega_{m}+\Omega_{\Lambda}=1$. In this case the expression for the luminosity distance has the integral form:

$$
d_{L}(z)=\frac{c}{H_{0}}(1+z) \int_{0}^{z} \frac{d z^{\prime}}{\left[\Omega_{m}\left(1+z^{\prime}\right)^{3}+\Omega_{\Lambda}\right]^{1 / 2}}
$$

An analytical fit to this expression, with a relative error of less than $0.4 \%$ for $0.2 \leq$ $\Omega_{m} \leq 1$, is presented in Pen (1999). In order to reduce the run time of our simulations, we have exploited this fit to the luminosity distance.

In a $\Lambda$-dominated cosmology, the number of GRBs per redshift unit is still given by equation (2), but in this case the expression for the comoving volume element is:

$$
\frac{d V}{d z}=\frac{c}{H_{0}} \frac{4 \pi d_{L}^{2}(z)}{(1+z)^{2}\left[\Omega_{m}(1+z)^{3}+\Omega_{\Lambda}\right]^{1 / 2}}
$$

For the GRB redshift distribution we adopt the same function as for the $\Lambda=0$ cosmology, with the same value of $z_{\max }$. To take into account the difference in luminosity density between an Einstein-de Sitter and a $\Lambda$ flat Universe, we applied to $n(z)$ the correction factor $\left[\Omega_{m}(1+z)^{3}+\Omega_{\Lambda}\right]^{1 / 2} /(1+z)^{3 / 2}$ (see the Appendix of Porciani \& Madau 2001 for details).

In order to study the ability of GRBs in probing the cosmological parameters in a $\Lambda$-dominated Universe, we have simulated $10^{2}$ realizations of GRB samples with $N_{G R B}$ $=10,30,100,300$ and 1000. The $\chi^{2}$ minimization of the resulting Hubble diagrams has been performed considering $\log d_{L}^{2}$ depending only on the fit parameter $\Omega_{m}$, i.e., using the relation $\Omega_{\Lambda}=1-\Omega_{m}$. Table 2 reports the general results of our repeated fits for a flat cosmology with input values $\Omega_{m}=0.3$ and $\Omega_{\Lambda}=0.7$ (which are those adopted in Frail et al. 2001).

As in the $\Lambda=0$ case, the accuracy of GRBs in constraining the two contributions $\Omega_{m}$ and $\Omega_{\Lambda}$ to the density parameter increases with their number $N_{G R B}$, reducing the dispersion about the best value for the fit parameter $\Omega_{m}$ from $\sim 40 \%$ for a sample with $N_{G R B}=10$ to $\sim 4 \%$ for $N_{G R B}=1000$. 


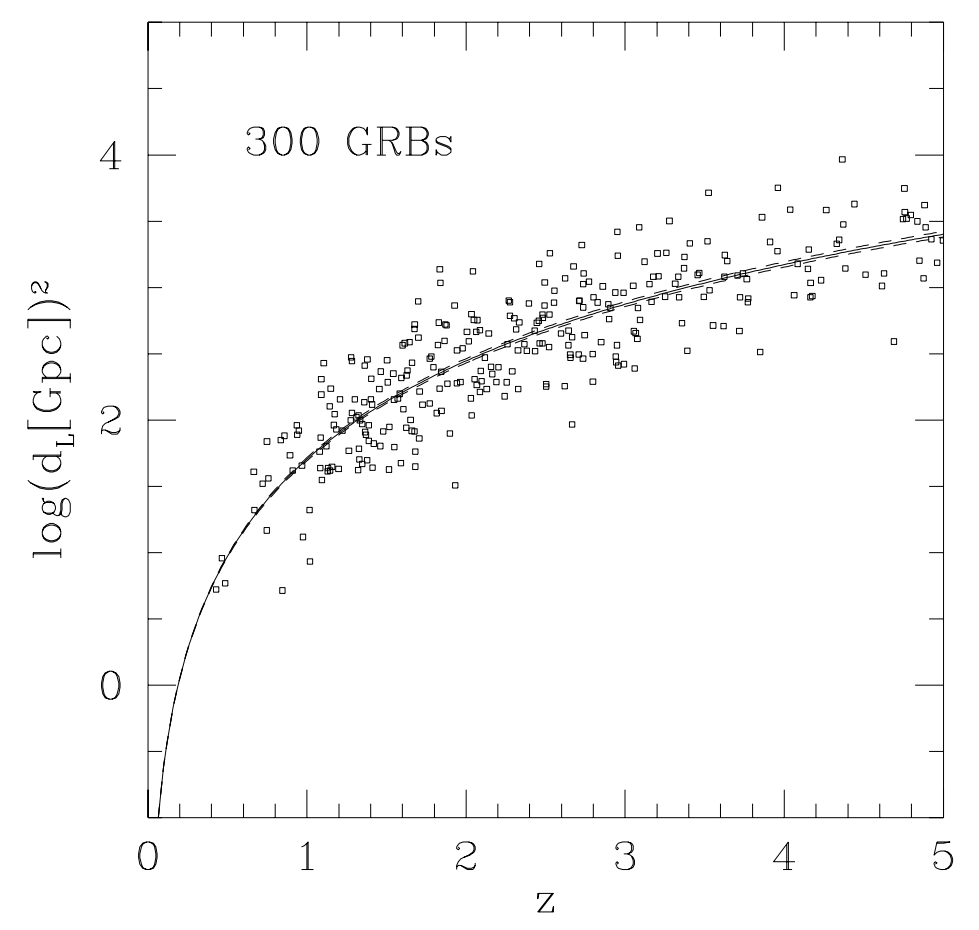

Figure 1. Hubble diagram $\log d_{L}^{2}-z$ with data simulated for a sample of 300 GRBs in a flat Universe with density parameters $\Omega_{m}=0.3$ and $\Omega_{\Lambda}=0.7$. The solid curve shows the function $\log d_{L}^{2}(z)$ in the assumed cosmology, while the dashed curves give the dispersion about the best fit parameter (the upper curve corresponds to lower $\Omega_{m}$ ).

Focussing on the samples in a $\Lambda$-dominated cosmology with $N_{G R B}=300$, a data set which can be realistically obtained in future space missions, Figure 1 shows one of the Hubble diagrams $\log d_{L}^{2}-z$ obtained with the simulations. The solid curve shows the function $\log d_{L}^{2}(z)$ in the assumed cosmology, while the dashed curves give the dispersion about the best fit parameters. The ability of a sample of 300 GRBs in constraining the actual cosmology is evident. The statistical fluctuations of the $N_{G R B}=300$ sample fit are outlined in the histogram of Figure 2. which shows the distribution of the best fit values of the matter density fraction $\Omega_{m}$ for $10^{3}$ sample realizations. The distribution peaks at $\Omega_{m}=0.3$, has a dispersion $S_{\Omega_{m}}=0.0228$, and a kurtosis $k_{\Omega_{m}}=3.0993$, to be compared with the value of a Gaussian distribution, i.e., 3.

We have also investigated the effects of changing the assumptions of our simulations on the probing ability of GRB samples to determine the geometry of the Universe. First, we have considered a GRB redshift distribution ruled by the simple function $n(z) \propto(1+z)^{3}$ instead of equation (44). The result is a slight decrease of the dispersion about the best fit parameter $\Omega_{m}$ at all values of $N_{G R B}$. This is due to the larger number of GRBs sampled at high redshift values by this alternative distibution, which increases monotonically with $z$. At high redshifts the distinction between different cosmologies becomes more evident (see curves in Figure 1), thus more GRBs at large $z$ imply better 


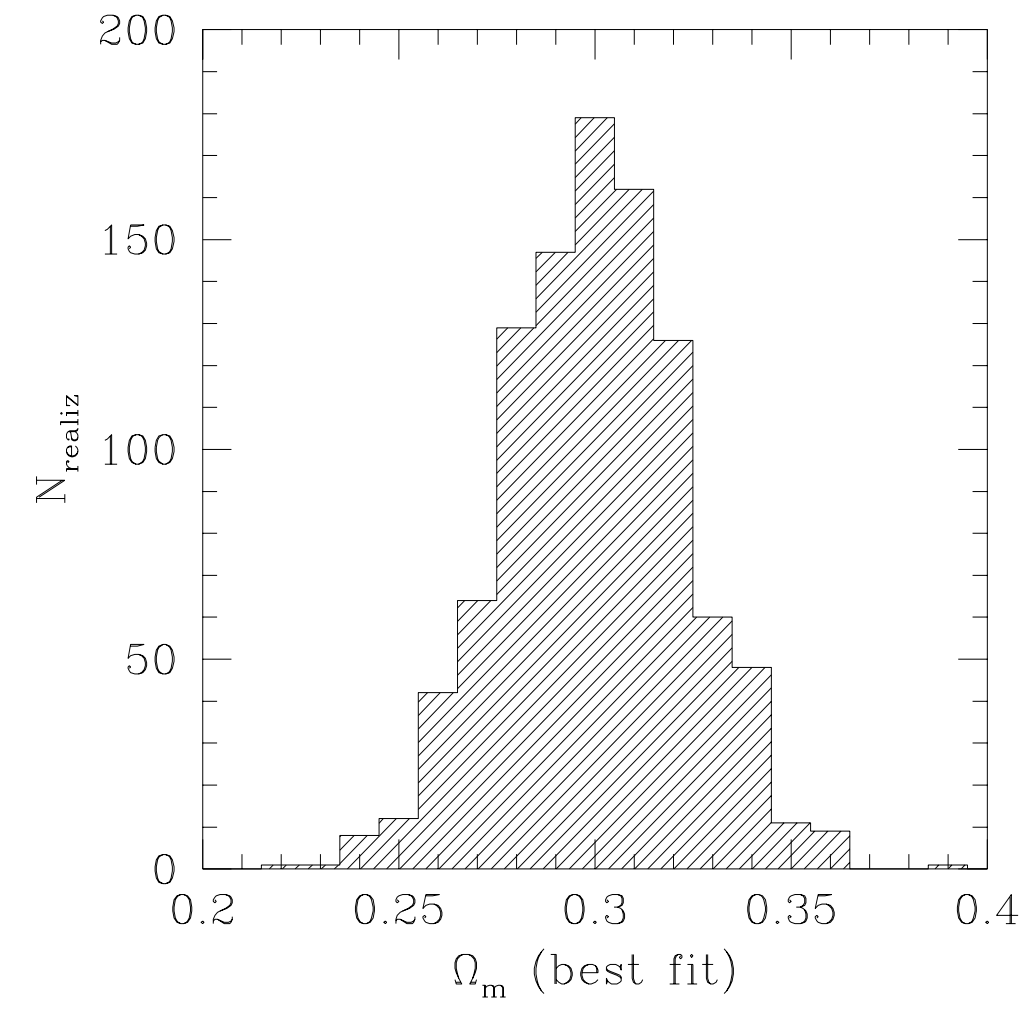

Figure 2. Histogram with the distribution of the best fit values of the matter density $\Omega_{m}$ for $10^{3}$ realizations of a sample of 300 GRBs in a flat Universe with density parameters $\Omega_{m}=0.3$ and $\Omega_{\Lambda}=0.7$. The distribution has a mean $\left\langle\Omega_{m}\right\rangle=0.3001$, a median $\Omega_{m}($ med $)=0.3002$, a dispersion $S_{\Omega_{m}}=0.0228$, and a kurtosis $k_{\Omega_{m}}=3.0993$.

constraints on the cosmological parameters.

Then we have studied the effect of varying the dispersion about the standard candle energy. We assumed $\sigma=0.6$ (doubling the dispersion to a multiplicative factor of 4) about the logarithmic mean value $\mu=51.1$ reported in Bloom, Frail, \& Kulkarni (2003). We find that the resulting effect is of course a worse accuracy in the reproduction of the input cosmology, the dispersion about the best fit parameter $\Omega_{m}$ increasing by a factor $\sim 2$ at all values of $N_{G R B}$. In particular, it is $\sim 15 \%$ for a sample with $N_{G R B}=300$.

Moreover, we point out that the variation of the standard candle energy $E_{\gamma}$ has no effect on the ability of GRB samples in putting constraints on cosmological parameters, since the mean value of the Gaussian distribution of $\log E_{\gamma}$ gives only a normalization constant to our simulations, but is not instructive for their scatter.

Finally, we must remark that the analyses of both Frail et al. (2001) and Bloom, Frail, \& Kulkarni (2003) assume of course a particular set of cosmological parameters $\left(\Omega_{m}=0.3, \Omega_{\Lambda}=0.7\right.$, and $\left.H_{0}=65 \mathrm{~km} \mathrm{~s}^{-1} \mathrm{Mpc}^{-1}\right)$ to derive the standard $\gamma$-ray energy of GRBs. To avoid a circular logic and the limitations in cosmographic applications pointed out in Bloom, Frail, \& Kulkarni (2003), we should assume a candle calibration with a local sample of sources, a prospect which can now be considered possible in the light of the discovery of the nearby GRBs 030329 and 031203 (see Section 5 for a more 
detailed discussion on this point).

\section{Simulations in a quintessence cosmology}

Now we abandon the cosmological constant Universe and we consider some of the most popular quintessence models. In particular, we choose as tracker potential classes the inverse power-law Ratra-Peebles potential (hereafter RP; Ratra \& Peebles 1988), defined as:

$$
V(\phi)=\frac{M^{4+\alpha}}{\phi^{\alpha}}
$$

and the SUGRA potential (Binétruy 1999; Brax \& Martin 1999):

$$
V(\phi)=\frac{M^{4+\alpha}}{\phi^{\alpha}} \exp \left(4 \pi G \phi^{2}\right) .
$$

Following Caresia, Matarrese, \& Moscardini (2004), we considered such potentials within the framework of "extended quintessence" models. These are characterized by a coupling between gravity and quintessence ruled by a parameter $\xi$, where $\xi=0$ means no coupling. By fixing $\alpha$ and $\xi$, we obtained numerical values for the luminosity distance $d_{L}(z)$ with the standard procedure. First, we numerically solved the Klein-Gordon equation for the scalar field $\phi$. From this solution it is possible to get the expansion rate $H(z)=H_{0} h(z)$, which depends on $\phi$ since the quintessence scalar field contributes to the total energy density. Then we exploited the usual relation between expansion rate and luminosity distance:

$$
d_{L}(z)=\frac{c}{H_{0}}(1+z) \int_{0}^{z} \frac{d z^{\prime}}{h\left(z^{\prime}\right)}
$$

We repeated the same procedure for the dilaton scenario introduced by Gasperini, Piazza, \& Veneziano (2002; hereafter GPV). In order to distinguish between different tracker potentials of the same class, say RP, we will use a couple of indices, the first one giving the value of the $\alpha$ parameter, while the second one referring to the adopted coupling parameter $\xi$, being 1 for $\xi=0$ and 2 for $\xi=0.01$ (therefore, model RP01 corresponds to no quintessence). In Figure 3 we report the function $\log d_{L}^{2}(z)$ found for the two most "extreme" among the quintessence models considered, i.e., those in which this function differs most strongly from that corresponding to a $\Lambda$-dominated flat Universe with density parameters $\Omega_{m}=0.3$ and $\Omega_{\Lambda}=0.7$, already shown in Figure 1 . All the other models give a $\log d_{L}^{2}(z)$ curve lying between the no quintessence case and the RP22 curve.

Figure 3 allows us to make an important, technical point. We have so far postponed the thorny issue of the calibration of the absolute energy release: in fact, when a sample of GRBs is used to derive the distribution of energy releases, it is necessary to assume a set of cosmological parameters to compare GRBs at different redshifts, making (potentially!) our argument circular. However, it is well known that, for $z \ll 1$, all cosmological models coincide, so that a local calibration of the absolute energy release 


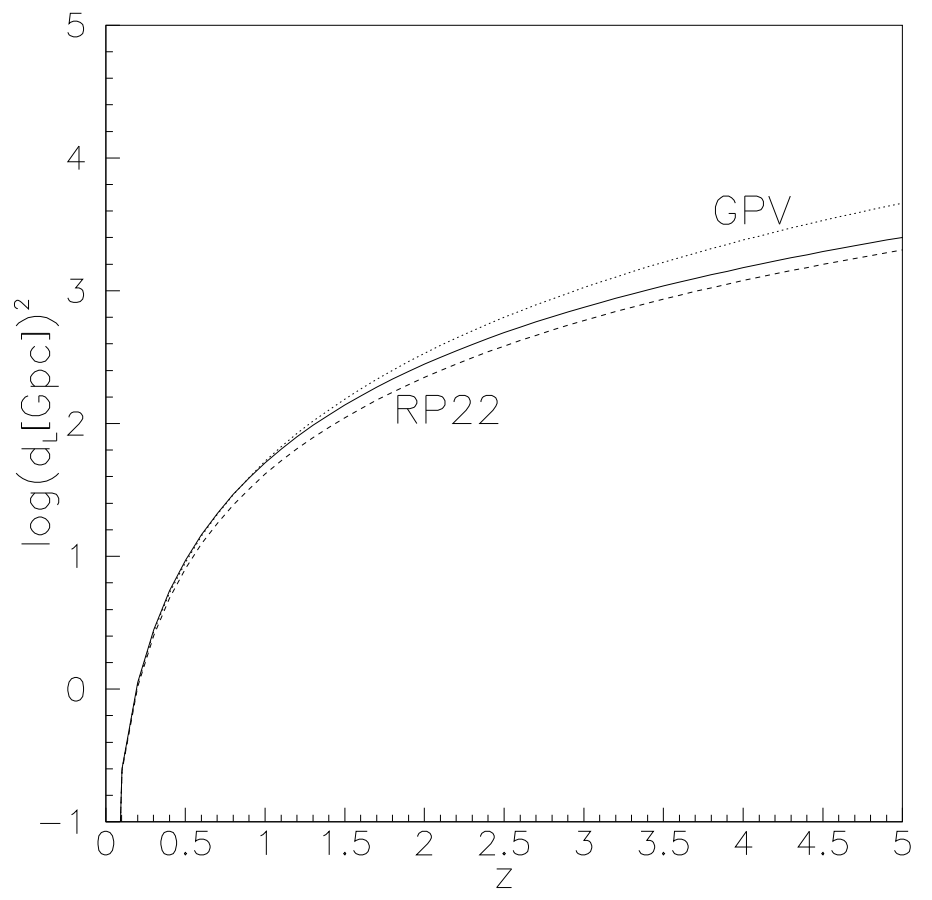

Figure 3. The parameter $\log d_{L}^{2}$ as a function of redshift $z$ in a flat Universe with density parameters $\Omega_{m}=0.3$ and $\Omega_{\Lambda}=0.7$ (solid curve) compared with those obtained in the quintessence models RP22 (lower dashed curve) and GPV (upper dotted curve).

is possible. Originally, given the very large redshifts of the first GRBs, it was not clear whether this could be achieved, but with the accumulation of further data this does not look like a real concern: there are currently 4 GRBs with $z<0.4$, out of a total of 39 (Figure 4), making a local calibration with a large sample of GRBs a real possibility.

Furthermore, the situation is even better if we assume $\Omega_{m}+\Omega_{\Lambda}=1$ : we see from Figure 3 that all models, including GPV which is by far the most discordant one, yield essentially the same luminosity distance out to $z \approx 1$. There are currently 17 GRBs with $\mathrm{z}<1$ out of a total of 39 (Figure 4), making the issue of calibration a moot one, once a sufficiently large sample is obtained.

In order to determine the number of GRBs per redshift unit in quintessence universes, we can exploit again the Appendix of Porciani \& Madau (2001) and find that:

$$
\frac{d N_{G R B}}{d z}=\frac{d_{L}^{2}(z) n(z) /(1+z)^{7 / 2}}{\int_{0}^{z_{\max }} d_{L}^{2}(z) n(z) /(1+z)^{7 / 2} d z}
$$

independently of $d V / d z$, where we have already taken into account the correction factor for the difference in luminosity density from the Einstein-de Sitter Universe. The adopted GRB redshift distribution $n(z)$ is always given by equation (4), and we fix again $z_{\max }=5$. 
Our purpose is now to investigate whether it is possible to discriminate between different quintessence cosmological models via a set of GRBs, considered as standard candles. To do this, we performed a series of KS tests on simulated data sets with $N_{G R B}$ $=100,300$ and 1000 in two different $\Lambda$-dominated flat cosmologies both with $\Omega_{m}=0.3$ and $\Omega_{\Lambda}=0.7$ at $z=0$, but, while one has a truly constant $\Lambda$, the other one has a quintessence field which reduces to $\Omega_{\Lambda}=0.7$ at $z=0$. The model for quintessence is chosen from those listed before. We considered both the case in which the same set of GRB redshifts is used, and the case in which different patches of the Universe are selected, each with its own cosmology, and thus two fully distinct sets of GRB redshifts are used for the two different cosmologies. In this second way, we wish to include the cosmic variance into our simulations. Our results are presented in Table 3, for a same GRB redshift distribution, and in Table 4, where cosmic variance has been considered.

From Tables 3 and 4 we see that it is quite difficult to discriminate a quintessence cosmological model using a set of up to 1000 GRBs as standard candles. Only the GPV model could be significantly discriminated with a set of 1000 GRBs, especially if we take into account cosmic variance, in which case we obtained $\left\langle Q_{K S}\right\rangle=4.89 \cdot 10^{-8}$. A discrimination may also be possible for the RP22 model, which resulted in a $<Q_{K S}>=8.45 \cdot 10^{-3}$ with $1000 \mathrm{GRBs}$, when not considering cosmic variance. These results are consistent with the extreme behaviours of the $\log d_{L}^{2}(z)$ functions for GPV and RP22 models, as already stated before and shown in Figure 3

These findings may be the consequence of the high dispersion, $\sigma=0.3$ in its logarithm, around the mean value of the geometrically corrected $\gamma$-ray energy released by GRBs, which we have taken as a cosmological candle. In order to investigate whether a better discrimination of different quintessence models could be obtained with a pin down of our standard candle, we performed another series of KS tests on simulated data sets, in which we adopted a SNIa-like candle with a logarithmic dispersion of $\sigma=0.072$, corresponding to a conservative magnitude dispersion of $\sigma_{M}=0.18 \mathrm{mag}$, intermediate between the $\sigma_{M}=0.21 \mathrm{mag}$ value found by Riess, Press, \& Kirshner (1995) and the later $\sigma_{M}=0.12 \mathrm{mag}$ of the same authors (Riess, Press, \& Kirshner 1996) together with the very recent results by Wang et al. (2003) in the range $\sigma_{M}=0.08-0.11 \mathrm{mag}$. The conditions of the simulations were the same as for the first KS test series, with the additional subdivision of a value $z_{\max }=5$, typical of GRBs, or 2, more typical of SNIa. In the first case, our results are reported in Table 5 , for the same standard candle redshift distribution, and in Table 6, where cosmic variance has been considered, while in the second case the results are shown respectively in Table 7 and Table 8 ,

Analysis of Tables 5-8 shows that with a less dispersed cosmological candle the GPV and RP22 quintessence models could be significantly discriminated with a set of 1000 sources observed up to $z_{\max }=5$, the first one in this case perhaps even with only 300 sources $\left(<Q_{K S}\right\rangle=1.04 \cdot 10^{-3}$ taking into account cosmic variance). Moreover, a hint for discrimination is also given with 1000 candles by the RP21 model, which resulted in a $\left\langle Q_{K S}>=6.92 \cdot 10^{-3}\right.$ when not considering cosmic variance. On the other hand, limiting the candle distribution to $z_{\max }=2$, it seems possible to significantly 
discriminate all the quintessence models of the RP tracker potential class, together with the GPV model, if 1000 sources were observed. The RP21 and RP22 models may be discriminated even with a data set of only 300 standard candles. These results are consistent with Figure 3, where it is possible to notice how up to $z=2$ the $\log d_{L}^{2}(z)$ function for the RP22 (and also for the not reported RP21) model differs more than the GPV one from the no quintessence case. There seems to be no chance for a discrimination of the SUGRA class of quintessence models, whose $\log d_{L}^{2}(z)$ functions are in fact very similar to the solide curve of Figure 3

\section{Conclusions}

We have simulated different samples of GRBs adopting $\gamma$-ray energy and redshift distributions consistent with recent observational results, in order to investigate their ability to probe cosmological parameters such as the density fractions $\Omega_{m}$ and $\Omega_{\Lambda}$. Our result is that in a $\Lambda$-dominated flat Universe the accuracy in the determination of the matter density $\Omega_{m}$ is $\sim 40 \%$ for a sample with $N_{G R B}=10$ and an excellent $\sim 4 \%$ for $N_{G R B}=1000$.

For comparison, a $\sim 20 \%$ accuracy on the determination of $\Omega_{m}$ has been recently claimed by using GRBs as standard candles ruled by the luminosity-variability and luminosity-lag time relations (Takahashi et al. 2003).

Since GRBs are much more readily observed than SNIa, especially at high redshifts (notice that in the sample observed so far, which is reported in Figure 4, there are already 4 GRBs with $z>3$, about $10 \%$ of the whole), they should allow us to probe cosmological parameters more deeply than these latter sources. Moreover, during the last few years of observations the number of GRBs with known redshifts has almost reached the same number of high redshift SNIa discovered by the Supernova Cosmology Project (Perlmutter et al. 1999).

Lastly, after showing that the absolute energy release can be calibrated using the low redshift GRBs, we have shown that GRBs have the potential to investigate the luminosity distance out to large redshifts, and this, in turn, means that at least some models for quintessence, among which the important dilaton model of Gasperini, Piazza and Veneziano, can be tested and discriminated from competing models.

On 20 November 2004, the Swift satellite was launched, and the detection of $\sim 200$ GRBs with known redshifts is expected during the 3 years of its sky observations. If GRBs are confirmed by these new data to emit a standard amount of energy, then our simulations stress how the accuracy in the determination of cosmological parameters increases with the number of their known redshifts, making Swift, at least potentially, a GRB Cosmology Project. 


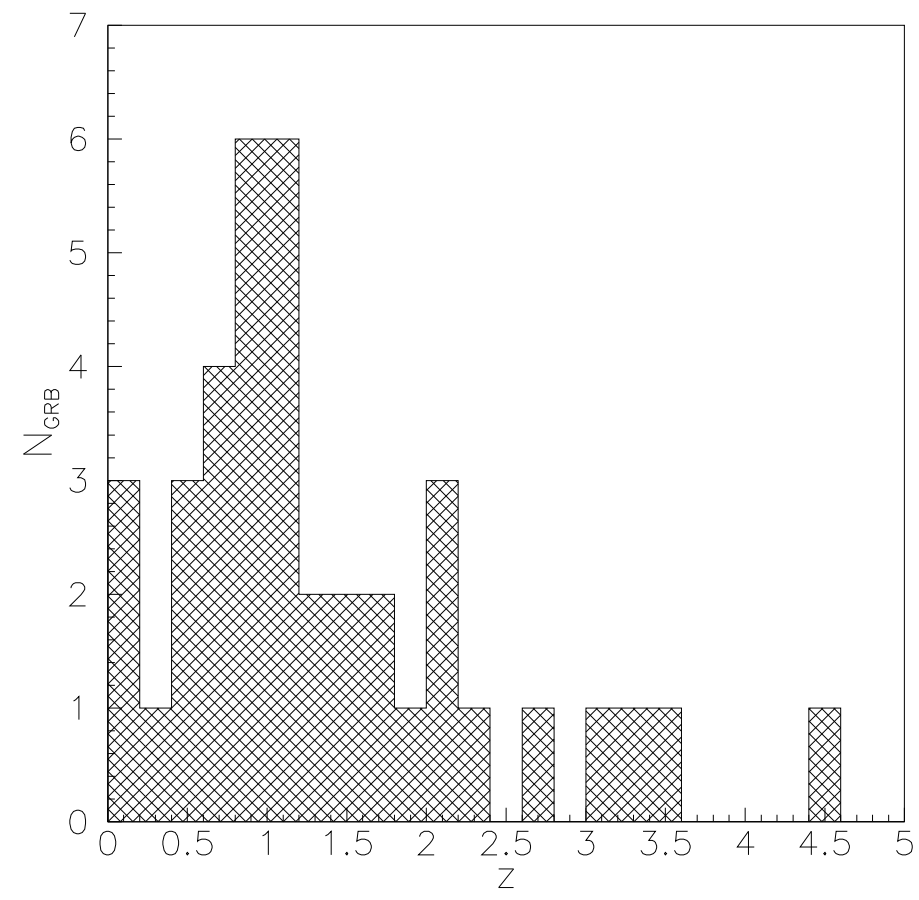

Figure 4. Distribution of all the known redshifts of GRBs as of November 2004. All redshifts are within the 0.1-4.5 range, apart from GRB 980425, possibly associated with the nearby SN1998bw ( $\mathrm{z}=0.0085$, Galama et al. 1998). Data is taken from Greiner (2004).

\section{References}

Bennett C L et al. 2003 ApJS 1481

Berger E, Kulkarni S R and Frail D A 2003 ApJ 590379

Binétruy P 1999 Phys. Rev. D 60063502

Bloom J S, Frail D A and Kulkarni S R 2003 ApJ 594674

Bloom J S, Frail D A and Sari R 2001 AJ 1212879

Brax P and Martin J 1999 Phys. Lett. B 46840

Caresia P, Matarrese S and Moscardini L 2004 ApJ 60521

Cohen E and Piran T 1997 ApJ 488 L7

Dai Z G, Liang E W and Xu D $2004 A p J 612$ L101

Frail D A et al. 2001 ApJ 562 L55

Galama T J et al. 1998 Nature 395670

Gasperini M, Piazza F and Veneziano G 2002 Phys. Rev. D 65023508 (GPV)

Ghirlanda G, Ghisellini G and Lazzati D 2004 ApJ 616331

Ghirlanda G, Ghisellini G, Lazzati D and Firmani C 2004 ApJ 613 L13

Greiner J 2004 GRB webpage, http://www.mpe.mpg.de/ jjcg/grbgen.html

Malesani D et al. $2004 A p J 609$ L5

Panaitescu A and Kumar P 2001 ApJ 560 L49

Pen U L 1999 ApJS 12049

Perlmutter S et al. 1999 ApJ 517565

Piran T et al. $2001 A p J 560$ L167

Porciani C and Madau P 2001 ApJ 548522 
Price P A et al. 2003 Nature 423844

Ratra B and Peebles P J E 1988 Phys. Rev. D 373406 (RP)

Riess A G, Press W H and Kirshner R P 1995 ApJ 438 L17

1996 ApJ 47388

Schaefer B E 2003 ApJ $\mathbf{5 8 3}$ L67

Takahashi K, Oguri M, Kotake K and Ohno H 2003 Preprint astro-ph/0305260

Von Mises R 1964 Mathematical Theory of Probability and Statistics (New York: Academic Press) Chapter IX

Wang L, Goldhaber G, Aldering G and Perlmutter S 2003 ApJ 590944 
Table 1. Mean values of the fitted cosmological density parameter $\Omega_{m}$, of its error $\Delta \Omega_{m}$ and its dispersion $S_{\Omega_{m}}$ obtained by fitting $10^{2}$ (top), $10^{3}$ (middle) and $10^{4}$ (bottom) GRB sample realizations with $N_{G R B}$ distributed according to function $R_{S F 1}(z)$ of Porciani \& Madau (2001) in an Einstein-de Sitter Universe $\left(\Omega_{m}=1\right)$.

\begin{tabular}{cccc}
\hline$N_{G R B}$ & $<\Omega_{m}>$ & $<\Delta \Omega_{m}>$ & $S_{\Omega_{m}}$ \\
\hline 10 & 0.9983 & 0.2997 & 0.3097 \\
30 & 1.0158 & 0.1895 & 0.1993 \\
100 & 0.9937 & 0.0993 & 0.1108 \\
300 & 0.9959 & 0.0599 & 0.0629 \\
1000 & 1.0009 & 0.0332 & 0.0351 \\
\hline & & & \\
\hline$N_{G R B}$ & $<\Omega_{m}>$ & $<\Delta \Omega_{m}>$ & $S_{\Omega_{m}}$ \\
\hline 10 & 1.0265 & 0.3095 & 0.3483 \\
30 & 1.0142 & 0.1766 & 0.1993 \\
100 & 1.0026 & 0.0993 & 0.1048 \\
300 & 1.0020 & 0.0593 & 0.0595 \\
1000 & 1.0015 & 0.0331 & 0.0333 \\
\hline & \multicolumn{3}{c}{} \\
\hline$N_{G R B}$ & $<\Omega_{m}>$ & $<\Delta \Omega_{m}>$ & $S_{\Omega_{m}}$ \\
\hline 10 & 1.0320 & 0.3085 & 0.3516 \\
30 & 1.0103 & 0.1780 & 0.1939 \\
100 & 1.0043 & 0.0997 & 0.1074 \\
300 & 1.0011 & 0.0590 & 0.0614 \\
1000 & 1.0004 & 0.0330 & 0.0332 \\
\hline
\end{tabular}

Table 2. Mean values of the fitted cosmological density parameters $\Omega_{m}$ and $\Omega_{\Lambda}$, of their error $\Delta \Omega$ and their dispersion $S_{\Omega}$ obtained by fitting $10^{2}$ GRB sample realizations with $N_{G R B}$ distributed according to function $R_{S F 1}(z)$ of Porciani \& Madau (2001) in a flat Universe with input values $\Omega_{m}=0.3$ and $\Omega_{\Lambda}=0.7$.

\begin{tabular}{ccccc}
\hline$N_{G R B}$ & $\left\langle\Omega_{m}\right\rangle$ & $\left\langle\Omega_{\Lambda}\right\rangle$ & $\langle\Delta \Omega\rangle$ & $S_{\Omega}$ \\
\hline 10 & 0.3195 & 0.6805 & 0.1004 & 0.1307 \\
30 & 0.2973 & 0.7027 & 0.0763 & 0.0700 \\
100 & 0.3002 & 0.6998 & 0.0363 & 0.0351 \\
300 & 0.3023 & 0.6977 & 0.0219 & 0.0222 \\
1000 & 0.3001 & 0.6999 & 0.0120 & 0.0125 \\
\hline
\end{tabular}


Table 3. Mean values of the probability $Q_{K S}$ and the maximum distance $D$ for a KS test on the parameter $\log d_{L}^{2}(z)$ of 100 realizations of a redshift sample made of 100 (top), 300 (middle) and 1000 (bottom) GRBs obtained in a $\Lambda$-dominated flat cosmology with $\Omega_{m}=0.3$ and $\Omega_{\Lambda}=0.7$ at $z=0$, but with two different $\log d_{L}^{2}(z)$ distributions, one resulting from a cosmology with a truly constant $\Lambda$ and the other from a quintessence model defined by its name in the first column, which reduces to the same cosmology at $z=0$.

\begin{tabular}{ccc}
\hline Model & $<Q_{K S}>$ & $<D>$ \\
\hline RP01 & $6.84 \cdot 10^{-1}$ & 0.098 \\
RP11 & $6.08 \cdot 10^{-1}$ & 0.107 \\
RP12 & $5.75 \cdot 10^{-1}$ & 0.110 \\
RP21 & $4.91 \cdot 10^{-1}$ & 0.120 \\
RP22 & $4.54 \cdot 10^{-1}$ & 0.125 \\
SUGRA11 & $7.29 \cdot 10^{-1}$ & 0.094 \\
SUGRA12 & $7.25 \cdot 10^{-1}$ & 0.094 \\
SUGRA21 & $6.37 \cdot 10^{-1}$ & 0.104 \\
SUGRA22 & $6.52 \cdot 10^{-1}$ & 0.103 \\
GPV & $2.76 \cdot 10^{-1}$ & 0.149 \\
\hline & & \\
\hline Model & $<Q_{K S}>$ & $<D>$ \\
\hline RP01 & $6.98 \cdot 10^{-1}$ & 0.057 \\
RP11 & $4.37 \cdot 10^{-1}$ & 0.073 \\
RP12 & $4.22 \cdot 10^{-1}$ & 0.076 \\
RP21 & $2.14 \cdot 10^{-1}$ & 0.094 \\
RP22 & $1.41 \cdot 10^{-1}$ & 0.102 \\
SUGRA11 & $6.38 \cdot 10^{-1}$ & 0.060 \\
SUGRA12 & $6.12 \cdot 10^{-1}$ & 0.061 \\
SUGRA21 & $5.05 \cdot 10^{-1}$ & 0.069 \\
SUGRA22 & $5.26 \cdot 10^{-1}$ & 0.067 \\
GPV & $8.32 \cdot 10^{-2}$ & 0.113 \\
\hline & & \\
\hline Model & $<Q_{K S}>$ & $<D>$ \\
\hline RP01 & $7.16 \cdot 10^{-1}$ & 0.031 \\
RP11 & $1.85 \cdot 10^{-1}$ & 0.053 \\
RP12 & $1.35 \cdot 10^{-1}$ & 0.056 \\
RP21 & $1.98 \cdot 10^{-2}$ & 0.076 \\
RP22 & $8.45 \cdot 10^{-3}$ & 0.082 \\
SUGRA11 & $4.70 \cdot 10^{-1}$ & 0.039 \\
SUGRA12 & $4.56 \cdot 10^{-1}$ & 0.040 \\
SUGRA21 & $3.19 \cdot 10^{-1}$ & 0.045 \\
SUGRA22 & $2.94 \cdot 10^{-1}$ & 0.047 \\
GPV & $3.28 \cdot 10^{-4}$ & 0.103 \\
\hline
\end{tabular}


Table 4. Mean values of the probability $Q_{K S}$ and the maximum distance $D$ for a KS test on the parameter $\log d_{L}^{2}(z)$ of 100 realizations of two different samples made of 100 (top), 300 (middle) and 1000 (bottom) GRBs, one obtained in a $\Lambda$-dominated flat cosmology with $\Omega_{m}=0.3$ and $\Omega_{\Lambda}=0.7$, and the other in a quintessence model defined by its name in the first column, which reduces to the same cosmology at $z=0$.

\begin{tabular}{ccc}
\hline Model & $<Q_{K S}>$ & $<D>$ \\
\hline RP01 & $4.64 \cdot 10^{-1}$ & 0.126 \\
RP11 & $4.91 \cdot 10^{-1}$ & 0.123 \\
RP12 & $4.91 \cdot 10^{-1}$ & 0.123 \\
RP21 & $4.60 \cdot 10^{-1}$ & 0.128 \\
RP22 & $3.55 \cdot 10^{-1}$ & 0.143 \\
SUGRA11 & $4.97 \cdot 10^{-1}$ & 0.121 \\
SUGRA12 & $4.92 \cdot 10^{-1}$ & 0.121 \\
SUGRA21 & $4.52 \cdot 10^{-1}$ & 0.127 \\
SUGRA22 & $5.42 \cdot 10^{-1}$ & 0.117 \\
GPV & $1.41 \cdot 10^{-1}$ & 0.190 \\
\hline & & \\
\hline Model & $<Q_{K S}>$ & $<D>$ \\
\hline RP01 & $5.01 \cdot 10^{-1}$ & 0.070 \\
RP11 & $3.45 \cdot 10^{-1}$ & 0.082 \\
RP12 & $4.16 \cdot 10^{-1}$ & 0.076 \\
RP21 & $2.30 \cdot 10^{-1}$ & 0.099 \\
RP22 & $1.80 \cdot 10^{-1}$ & 0.106 \\
SUGRA11 & $5.16 \cdot 10^{-1}$ & 0.069 \\
SUGRA12 & $5.06 \cdot 10^{-1}$ & 0.070 \\
SUGRA21 & $4.18 \cdot 10^{-1}$ & 0.077 \\
SUGRA22 & $4.50 \cdot 10^{-1}$ & 0.074 \\
GPV & $7.36 \cdot 10^{-3}$ & 0.173 \\
\hline & & \\
\hline Model & $<Q_{K S}>$ & $<D>$ \\
\hline RP01 & $5.31 \cdot 10^{-1}$ & 0.037 \\
RP11 & $2.32 \cdot 10^{-1}$ & 0.053 \\
RP12 & $1.49 \cdot 10^{-1}$ & 0.062 \\
RP21 & $5.43 \cdot 10^{-2}$ & 0.077 \\
RP22 & $1.81 \cdot 10^{-2}$ & 0.086 \\
SUGRA11 & $3.74 \cdot 10^{-1}$ & 0.045 \\
SUGRA12 & $4.09 \cdot 10^{-1}$ & 0.043 \\
SUGRA21 & $3.54 \cdot 10^{-1}$ & 0.047 \\
SUGRA22 & $2.66 \cdot 10^{-1}$ & 0.051 \\
\hline & $4.89 \cdot 10^{-8}$ & 0.158 \\
\hline & &
\end{tabular}


Table 5. The same as Table 3 but obtained by adopting SNIa-like standard candles distributed with a logarithmic dispersion $\sigma=0.072$ up to $z_{\max }=5$.

\begin{tabular}{ccc}
\hline Model & $<Q_{K S}>$ & $<D>$ \\
\hline RP01 & $9.66 \cdot 10^{-1}$ & 0.062 \\
RP11 & $7.83 \cdot 10^{-1}$ & 0.090 \\
RP12 & $7.20 \cdot 10^{-1}$ & 0.096 \\
RP21 & $5.45 \cdot 10^{-1}$ & 0.112 \\
RP22 & $4.27 \cdot 10^{-1}$ & 0.125 \\
SUGRA11 & $9.21 \cdot 10^{-1}$ & 0.070 \\
SUGRA12 & $8.99 \cdot 10^{-1}$ & 0.075 \\
SUGRA21 & $8.52 \cdot 10^{-1}$ & 0.081 \\
SUGRA22 & $8.21 \cdot 10^{-1}$ & 0.086 \\
GPV & $1.93 \cdot 10^{-1}$ & 0.157 \\
\hline & & \\
\hline Model & $<Q_{K S}>$ & $<D>$ \\
\hline RP01 & $9.68 \cdot 10^{-1}$ & 0.037 \\
RP11 & $5.11 \cdot 10^{-1}$ & 0.067 \\
RP12 & $4.45 \cdot 10^{-1}$ & 0.071 \\
RP21 & $1.65 \cdot 10^{-1}$ & 0.092 \\
RP22 & $1.09 \cdot 10^{-1}$ & 0.099 \\
SUGRA11 & $8.19 \cdot 10^{-1}$ & 0.050 \\
SUGRA12 & $8.18 \cdot 10^{-1}$ & 0.050 \\
SUGRA21 & $6.59 \cdot 10^{-1}$ & 0.059 \\
SUGRA22 & $6.39 \cdot 10^{-1}$ & 0.060 \\
GPV & $2.03 \cdot 10^{-2}$ & 0.129 \\
\hline & & \\
\hline Model & $<Q_{K S}>$ & $<D>$ \\
\hline RP01 & $9.68 \cdot 10^{-1}$ & 0.020 \\
RP11 & $1.39 \cdot 10^{-1}$ & 0.052 \\
RP12 & $9.51 \cdot 10^{-2}$ & 0.056 \\
RP21 & $6.92 \cdot 10^{-3}$ & 0.076 \\
RP22 & $1.54 \cdot 10^{-3}$ & 0.087 \\
SUGRA11 & $5.49 \cdot 10^{-1}$ & 0.036 \\
SUGRA12 & $5.17 \cdot 10^{-1}$ & 0.037 \\
SUGRA21 & $2.95 \cdot 10^{-1}$ & 0.044 \\
GUGRA22 & $2.45 \cdot 10^{-1}$ & 0.046 \\
\hline & $5.57 \cdot 10^{-6}$ & 0.118 \\
\hline & &
\end{tabular}


Table 6. The same as Table 4 but obtained by adopting SNIa-like standard candles distributed with a logarithmic dispersion $\sigma=0.072$ up to $z_{\max }=5$.

\begin{tabular}{ccc}
\hline Model & $<Q_{K S}>$ & $<D>$ \\
\hline RP01 & $5.25 \cdot 10^{-1}$ & 0.118 \\
RP11 & $4.81 \cdot 10^{-1}$ & 0.122 \\
RP12 & $4.55 \cdot 10^{-1}$ & 0.127 \\
RP21 & $4.06 \cdot 10^{-1}$ & 0.138 \\
RP22 & $3.36 \cdot 10^{-1}$ & 0.145 \\
SUGRA11 & $4.59 \cdot 10^{-1}$ & 0.126 \\
SUGRA12 & $4.81 \cdot 10^{-1}$ & 0.121 \\
SUGRA21 & $4.66 \cdot 10^{-1}$ & 0.125 \\
SUGRA22 & $4.68 \cdot 10^{-1}$ & 0.128 \\
GPV & $6.28 \cdot 10^{-2}$ & 0.217 \\
\hline & & \\
\hline Model & $<Q_{K S}>$ & $<D>$ \\
\hline RP01 & $5.07 \cdot 10^{-1}$ & 0.069 \\
RP11 & $3.56 \cdot 10^{-1}$ & 0.083 \\
RP12 & $3.21 \cdot 10^{-1}$ & 0.088 \\
RP21 & $1.97 \cdot 10^{-1}$ & 0.101 \\
RP22 & $1.09 \cdot 10^{-1}$ & 0.118 \\
SUGRA11 & $4.52 \cdot 10^{-1}$ & 0.075 \\
SUGRA12 & $3.92 \cdot 10^{-1}$ & 0.079 \\
SUGRA21 & $4.14 \cdot 10^{-1}$ & 0.078 \\
SUGRA22 & $3.38 \cdot 10^{-1}$ & 0.083 \\
GPV & $1.04 \cdot 10^{-3}$ & 0.191 \\
\hline & & \\
\hline Model & $<Q_{K S}>$ & $<D>$ \\
\hline RP01 & $5.22 \cdot 10^{-1}$ & 0.038 \\
RP11 & $1.57 \cdot 10^{-1}$ & 0.059 \\
RP12 & $1.27 \cdot 10^{-1}$ & 0.063 \\
RP21 & $1.89 \cdot 10^{-2}$ & 0.082 \\
RP22 & $1.58 \cdot 10^{-2}$ & 0.089 \\
SUGRA11 & $3.58 \cdot 10^{-1}$ & 0.045 \\
SUGRA12 & $3.62 \cdot 10^{-1}$ & 0.046 \\
SUGRA21 & $2.27 \cdot 10^{-1}$ & 0.053 \\
SUGRA22 & $2.43 \cdot 10^{-1}$ & 0.051 \\
GPV & $6.07 \cdot 10^{-10}$ & 0.177 \\
\hline
\end{tabular}


Table 7. The same as Table 3 but obtained by adopting SNIa-like standard candles distributed with a logarithmic dispersion $\sigma=0.072$ up to $z_{\max }=2$.

\begin{tabular}{ccc}
\hline Model & $<Q_{K S}>$ & $<D>$ \\
\hline RP01 & $8.78 \cdot 10^{-1}$ & 0.077 \\
RP11 & $3.75 \cdot 10^{-1}$ & 0.131 \\
RP12 & $2.99 \cdot 10^{-1}$ & 0.141 \\
RP21 & $8.10 \cdot 10^{-2}$ & 0.188 \\
RP22 & $6.73 \cdot 10^{-2}$ & 0.192 \\
SUGRA11 & $7.14 \cdot 10^{-1}$ & 0.096 \\
SUGRA12 & $6.61 \cdot 10^{-1}$ & 0.101 \\
SUGRA21 & $5.55 \cdot 10^{-1}$ & 0.113 \\
SUGRA22 & $5.12 \cdot 10^{-1}$ & 0.116 \\
GPV & $3.33 \cdot 10^{-1}$ & 0.140 \\
\hline & & \\
\hline Model & $<Q_{K S}>$ & $<D>$ \\
\hline RP01 & $8.89 \cdot 10^{-1}$ & 0.044 \\
RP11 & $7.51 \cdot 10^{-2}$ & 0.108 \\
RP12 & $5.24 \cdot 10^{-2}$ & 0.116 \\
RP21 & $2.32 \cdot 10^{-3}$ & 0.157 \\
RP22 & $5.57 \cdot 10^{-4}$ & 0.176 \\
SUGRA11 & $4.56 \cdot 10^{-1}$ & 0.071 \\
SUGRA12 & $4.33 \cdot 10^{-1}$ & 0.072 \\
SUGRA21 & $2.19 \cdot 10^{-1}$ & 0.089 \\
SUGRA22 & $1.80 \cdot 10^{-1}$ & 0.092 \\
GPV & $4.93 \cdot 10^{-2}$ & 0.116 \\
\hline & & \\
\hline Model & $<Q_{K S}>$ & $<D>$ \\
\hline RP01 & $8.77 \cdot 10^{-1}$ & 0.025 \\
RP11 & $1.03 \cdot 10^{-3}$ & 0.094 \\
RP12 & $2.90 \cdot 10^{-4}$ & 0.100 \\
RP21 & $5.57 \cdot 10^{-8}$ & 0.143 \\
RP22 & $1.53 \cdot 10^{-9}$ & 0.158 \\
SUGRA11 & $1.10 \cdot 10^{-1}$ & 0.057 \\
SUGRA12 & $1.04 \cdot 10^{-1}$ & 0.057 \\
SUGRA21 & $1.85 \cdot 10^{-2}$ & 0.072 \\
GUGRA22 & $1.22 \cdot 10^{-2}$ & 0.076 \\
GPV & $1.25 \cdot 10^{-4}$ & 0.104 \\
\hline
\end{tabular}


Table 8. The same as Table 4 but obtained by adopting SNIa-like standard candles distributed with a logarithmic dispersion $\sigma=0.072$ up to $z_{\max }=2$.

\begin{tabular}{ccc}
\hline Model & $<Q_{K S}>$ & $<D>$ \\
\hline RP01 & $4.94 \cdot 10^{-1}$ & 0.122 \\
RP11 & $2.89 \cdot 10^{-1}$ & 0.153 \\
RP12 & $3.09 \cdot 10^{-1}$ & 0.150 \\
RP21 & $1.50 \cdot 10^{-1}$ & 0.189 \\
RP22 & $1.01 \cdot 10^{-1}$ & 0.199 \\
SUGRA11 & $4.53 \cdot 10^{-1}$ & 0.128 \\
SUGRA12 & $3.92 \cdot 10^{-1}$ & 0.135 \\
SUGRA21 & $3.82 \cdot 10^{-1}$ & 0.139 \\
SUGRA22 & $3.52 \cdot 10^{-1}$ & 0.142 \\
GPV & $2.42 \cdot 10^{-1}$ & 0.166 \\
\hline & & \\
\hline Model & $<Q_{K S}>$ & $<D>$ \\
\hline RP01 & $4.81 \cdot 10^{-1}$ & 0.070 \\
RP11 & $1.23 \cdot 10^{-1}$ & 0.116 \\
RP12 & $8.68 \cdot 10^{-2}$ & 0.117 \\
RP21 & $8.56 \cdot 10^{-3}$ & 0.162 \\
RP22 & $2.67 \cdot 10^{-3}$ & 0.179 \\
SUGRA11 & $3.27 \cdot 10^{-1}$ & 0.084 \\
SUGRA12 & $3.41 \cdot 10^{-1}$ & 0.084 \\
SUGRA21 & $2.30 \cdot 10^{-1}$ & 0.097 \\
SUGRA22 & $2.19 \cdot 10^{-1}$ & 0.098 \\
GPV & $3.89 \cdot 10^{-2}$ & 0.138 \\
\hline & & \\
\hline Model & $<Q_{K S}>$ & $<D>$ \\
\hline RP01 & $5.38 \cdot 10^{-1}$ & 0.037 \\
RP11 & $6.83 \cdot 10^{-3}$ & 0.097 \\
RP12 & $1.11 \cdot 10^{-3}$ & 0.103 \\
RP21 & $1.32 \cdot 10^{-7}$ & 0.149 \\
RP22 & $1.63 \cdot 10^{-8}$ & 0.164 \\
SUGRA11 & $1.44 \cdot 10^{-1}$ & 0.059 \\
SUGRA12 & $1.32 \cdot 10^{-1}$ & 0.061 \\
SUGRA21 & $3.56 \cdot 10^{-2}$ & 0.077 \\
GUGRA22 & $3.49 \cdot 10^{-2}$ & 0.079 \\
GPV & $5.27 \cdot 10^{-5}$ & 0.124 \\
\hline
\end{tabular}

